\title{
Bolonia 'for dummies'
}

\author{
Javier Diaz $^{1}$, Christian Morillas ${ }^{1}$, Samuel Romero ${ }^{1}$, Alberto Guillén ${ }^{1}$ \\ ${ }^{1}$ Dpto. Arquitectura y Tecnología de Granada. ETSIIT \\ Granada, España \\ $\{$ jiaz, cmorillas, sromero, aguillen\}@atc.ugr.es
}

\begin{abstract}
Resumen. El objeto de este trabajo es describir de forma sencilla y clara cómo impartir una asignatura nueva o transformar una asignatura existente, y la metodología docente para su impartición en el marco del Espacio Europeo de Educación Superior (EEES). Hacemos especial hincapié en aquellos aspectos afines a las materias impartidas tradicionalmente en Ingenierías como Informática, Telecomunicaciones o Electrónica, titulaciones relevantes en el área de Arquitectura y Tecnología de Computadores. Enunciamos los principios básicos y el enfoque que motiva y dirige la reforma educativa de Bolonia. Consecuencia de ello, describimos métodos sencillos para transformarnos en unos "nuevos docentes," alineados con la reforma educativa, tratando a la vez de minimizar el impacto que pueda tener en el profesor y de incluir nuevos enfoques de realización de nuestras tareas docentes.
\end{abstract}

Palabras Clave: Reforma educativa, EEES, Bolonia.

\section{Introducción}

"los cursos no valen..., si quiero aprender algo me leo un libro... y hago experimentos... ...deberíamos olvidarnos de la escuela y la universidad,... los niños deberían empezar el doctorado a los 5 años y que dure 20 años..."

Sydney Brenner (Premio Nobel de Medicina 2002). Redes (RTVE, Enero 2005)

"El monólogo frontal en que consiste la clase no sólo refuerza la pasividad del oyente, sino, más grave, suprime el espacio para cuestionar y cuestionarse, que es, justamente, lo que caracteriza a la docencia que entronque con la investigación. La labor del profesor no radica en transmitir los conocimientos adquiridos, en el mejor de los casos en la investigación más reciente, sino en enseñar a preguntar, orientando el trabajo y promoviendo el desarrollo intelectual y científico de los alumnos, lo que únicamente cabe en un diálogo personal"

Ignacio Sotelo, catedrático excedente de Sociología, (El País, 2 Febrero 2005) 
Este tipo de declaraciones ilustran cuál es el espíritu del que se nutre la Declaración de Bolonia de 1999, suscrita por 30 estados europeos no sólo de la UE sino países del Espacio Europeo de libre comercio y países del Este y Centro de Europa.

La reforma educativa asociada a la declaración de Bolonia o EEES es hoy en día una realidad (para pesadilla de algunos y deleite de otros). Por ejemplo, en el marco de la ETSIIT de la Universidad de Granada este año se está impartiendo el primero de los cursos de grado de las titulaciones ofertadas en la escuela (Ingeniería Informática y Telecomunicaciones). Pese a que "ya está aquí", muchos profesores no conocen las nuevas metodologías docentes necesarias para afrontar este proceso y no está claro cómo afectarán a su metodología docente habitual. Dado que a nivel institucional no se está dando formación para el profesorado, éste debe ser responsable e intentar adaptarse lo mejor posible haciendo un aprendizaje cooperando y coordinándose con otros colegas. En esa línea, este documento nace de la participación de algunos de los autores en el Curso de Iniciación a la Docencia impartido en la Universidad de Granada en 2010 [1]. Con él pretendemos, de forma muy básica, ofrecer un resumen de diferentes técnicas y protocolos de actuación convenientes para afrontar esa adaptación del profesorado y de sus asignaturas al EEES de modo que pueda servir de apoyo a quién lo estime oportuno.

Como veremos a lo largo de esta contribución, no se trata de cambiar todo, sino de concretar y cuantificar el esfuerzo del alumno en nuestra materia para conseguir los objetivos que tenemos, y diseñar métodos de aprendizaje que sustituyan las clases magistrales (de modo que se adquieran capacidades y no sólo conocimiento teórico).

Múltiples aspectos y enfoques son vitales para comprender el reto con el que nos encontramos. Entre ellos podemos destacar un enfoque del aprendizaje basado en el alumno y en la adquisición de competencias profesionales (donde dejamos sin respuesta la pregunta de si un profesor de universidad sabe realmente de ello), incorporación de una pluralidad de recursos didácticos y metodológicos y la incorporación clave del proceso de planificación detallada de los contenidos ( $\mathrm{y}$ temporización) de las distintas materias.

Notemos que los autores sólo pretenden mostrar su experiencia, parcial e incompleta, pero que esperamos pueda ayudar a otros compañeros perdidos como nosotros en esta vorágine de nuevos conceptos, metodologías y nuevos marcos legales y educativos.

Este trabajo está estructurado como sigue: en la Sección 2 damos una revisión de los aspectos principales que rigen el proceso EEES y que servirán como fundamentación del porqué y cómo cambiar nuestra metodología docente. En la sección 3 veremos el proceso de búsqueda de información marco para impartir una asignatura en los nuevos planes de grado. La sección 4 se encargará de mostrar algunos ejemplos de los recursos metodológicos que debemos utilizar para impartir dichas asignaturas. Finalmente, la sección 5 se dedicará a la discusión y conclusiones finales de este trabajo. 


\section{El espíritu de Bolonia ¿Qué quiere Bolonia de nosotros?}

Siguiendo la comunicación [9], debemos entender primero qué es (y qué no es) la reforma educativa promovida por la declaración de Bolonia.

¿QUÉ ES (o debería ser) EL ESPACIO EUROPEO DE EDUCACIÓN SUPERIOR?

A. Una oportunidad de mejora de la calidad de la docencia universitaria

B. Un cambio de paradigma: de un sistema basado en la enseñanza del profesor a un sistema basado en el aprendizaje del estudiante

C. Transparencia: contrato profesor-alumno (crédito ECTS como método de incluir el trabajo del alumno fuera del aula)

D. Una apuesta por una formación integral: contenidos científicos y capacidades (estudiantes como profesionales y ciudadanos)

¿QUÉ NO ES (o no debería ser) EL EEES?

A. Un cambio administrativo en la forma de medir la dedicación de los alumnos (¿y los profesores ¿quién y cómo nos evalúan?)

B. La obligación de preparar una tabla de equivalencias entre horas de clase (crédito tradicional) y horas de trabajo del alumno (crédito ECTS)

C. Crear nuevas víctimas del sistema:

a. Profesor: convertir una asignatura en una "gymkana de capacidades": "ahora tengo que enseñarles ciencias y, además, inglés, informática, a trabajar en grupo, habilidades de comunicación,..."

b. Alumno: la "oportunidad" para recibir menos clases y que los alumnos lo hagan todo por su cuenta

Como se desprende del análisis anterior, el proceso de convergencia europea pretender modificar significativamente (si no revolucionar) los objetivos y métodos docentes denominados clásicos. Por supuesto que muchos profesores ya trabajaban según esta metodología pero ahora se pretende desarrollarla de forma institucional y a nivel de toda Europa. Los principales aspectos de la reforma son:

1. Formación en base a competencias. Esto implica que se pretende dar un enfoque más aplicado al conocimiento. Un ejemplo de esto podría ser el siguient e:

En el marco de un examen de Ingeniero de Caminos, Canales y Puertosl, asignatura sobre materiales, solicitarle que realice el diseño de una estructura de hormigón de un puente concreto frente a una pregunta que describiera las propiedades del hormigón es un ejemplo de la orientación a competencias.

Este caso muestra que se le daría mayor importancia al saber hacer (diseñar la estructura usando hormigón del tipo adecuado y demás parámetros de diseño) que al saber (conocer las propiedades mecánicas y físico-químicas del hormigón). Información sobre las competencias podemos encontrarlas en:

- Genéricas (informe Tuning [5])

- Específicas (Libro Blanco [11])

- Titulación (documento VERIFICA [8])

A partir de esta idea, cada asignatura es formulada basada en competencias (qué sabrá hacer el estudiante tras finalizar la asignatura). Es importante destacar que cada 
competencia que se incluya deberá ser evaluada, luego habrá que ser cuidadosos para desarrollar un sistema de evaluación que permita asegurar que toda competencia ha sido cubierta y verificada.

Además, notemos que una misma competencia puede (y en muchos casos debe) estar cubierta por varias asignaturas y que, en teoría, podría ocurrir que una asignatura básica y muy necesaria para una titulación no tuviera competencia alguna asociada a la misma. Si bien este último caso es posible, la implantación de los planes de estudio ha forzado que todas las asignaturas tuvieran competencias (algunas veces se ha intentado que una asignatura/materia tenga más competencias con la idea que por ello sería más importante o que sin competencias pudiera desaparecer). Esperemos que en sucesivas modificaciones de los planes este problema desaparezca y las competencias se adecúen realmente a los contenidos que deben impartir las distintas materias.

Queremos destacar que el enfoque inicial basado en competencias profesionales (para saber hacer algo) ha sido modificado para incluir otras capacidades del estudiante de índole intelectual (capacidades de síntesis, análisis, expresión escrita) o de carácter social (actitudes frente a compañeros, capacidades de trabajo en grupo, etc.). Hemos pasado de este enfoque a uno en el que todo se denomina competencias (competencias instrumentales, competencias interpersonales, competencias sistémicas, etc.), independientemente de que tengan relación con el ejercicio de su carrera profesional o no lo que para muchos expertos carece de sentido. Esta discusión queda fuera del alcance de este documento y es más propia de profesionales del área de la didáctica y pedagogía pero es el origen de la "extraña" terminología usada en muchos documentos.

Finalmente, comentar que estas competencias (independientemente del tipo que sean), han sido divididas en los grados en dos apartados, competencias específicas del título y competencias transversales (estas últimas más asociadas a capacidades y sin referente como competencias directas profesionales).

En fin, mucha información en muy poco espacio. Para el lector que quiera ampliar le recomendamos la lectura de $[3,12,13]$.

2. Enfoque del proceso de aprendizaje basado en el estudiante. Para ello se deben usar nuevas metodologías docentes: desde herramientas para el aprendizaje autónomo, aprendizaje por indagación (guidado por el profesor pero realizado por el estudiante), colaborativo (en grupos) o la utilización de la clase magistral (pero con la inclusión de elementos que la hagan dinámica, evitando el mero "profesor habla, alumno escucha". Todos estos elementos requieren el conocimiento y la utilización de múltiples recursos docentes, así como el desarrollo de métodos de evaluación capaces no sólo de determinar el nivel de competencia adquirido por el alumno sino, además, de ayudar en el mismo proceso de aprendizaje. La tutoría se reinventa como método para ayudar a los estudiantes en el proceso de aprendizaje.

3. Nuevas maneras de planificar: uso de las guías didácticas. Estos nuevos objetivos que se le imponen al profesorado sólo son posibles mediante una planificación minuciosa de las clases, identificando qué tipo de contenido y actividades será conveniente abordar en cada una de ellas. Pese a la aparente rigidez de planificar, no debemos sentirlo de manera negativa ya que el objetivo es que sirva de guía y, en base a esa pluralidad de recursos docentes que deberemos poseer, 
deberíamos ser capaces de manejar flexiblemente esa planificación. Para más información sobre el proceso de planificación leer [7]. Todo ello se complementa y plasma en el uso de las guías didácticas. Estos documentos irán orientados al alumno (no al profesor como ocurre en el proyecto docente) y deberán ser capaces de guiar a alumno en el mar de actividades y recursos que debe utilizar para conseguir las competencias de la materia en curso. La Universidad de Granada realiza cursos específicos sobre elaboración de Guías didácticas y tiene muchos de los resultados de estos curso disponibles online. Como referencia ver la página web del Vicerrectorado de calidad [2] y como ejemplos consultar [4].

4. Finalmente, destacar que uno de los objetivos del EEES es la movilidad de estudiantes, ya sea horizontal (por ejemplo mediante el programa Erasmus) o vertical (mediante la convalidación de titulaciones). Ello se consigue mediante la utilización de "formatos de títulos comunes" (dos ciclos, uso de créditos ECTS, promoción de la movilidad, cooperaciones entre instituciones, suplemento europeo al título, etc.). El análisis de la consecución de este objetivo queda fuera del alcance de este trabajo aunque, como primera aproximación parece que se ha avanzado en los formatos de las titulaciones (grado + máster) y en consideraciones metodológicas de la docencia universitaria, quedando pendiente el hecho que las titulaciones tengan contenidos similares (que es un elemento clave para facilitar la movilidad de los estudiantes).

El resultado de todo este proceso es que los profesores universitarios deberán convertirse en verdaderos expertos en todo tipo de técnicas de comunicación, manejo de herramientas TIC, técnicas psicopedagógicas (dinamización de grupos, motivación del estudiante), etc. además de conocer adecuadamente su materia y aspectos de la misma relativos a las salidas profesionales que tuviera asociada. Pasar de una metodología básicamente magistral a este nuevo enfoque es, sin formación ni ayuda externa, una tarea ingente para el profesorado. Esperemos que con estas líneas podamos dar una pequeña "guía de bolsillo" que nos permita empezar con el proceso. Como lecturas complementarias sugerimos $[14,6]$.

\section{3 ¿Cómo desarrollo los contenidos de mi asignatura en el grado?}

Ya sea porque nos ha tocado impartir una nueva asignatura o bien porque tenemos que "adaptar" una parecida que dábamos en el plan antiguo, debemos prepararnos para esta nueva forma de impartir las clases. Si miramos el marco legislativo, hemos dejado atrás la Ley de la Reforma Universitaria (LRU) y por tanto los contenidos de las asignaturas que venían como descriptores en el BOE carecen actualmente de validez en los nuevos planes. El marco legislativo actual viene dado por la Ley Orgánica 6/2001 de 21 de diciembre, de Universidades (LOU), modificada en la Ley Orgánica 4/2007 de 12 de abril. A nivel local deberemos atender a la Ley 15/2003, de 22 de diciembre, Andaluza de Universidades (LAU) y a la legislación interna de la Universidad de Granada. Información relativa al marco legal LOU/LAU puede ser encontrada en la Tabla 1. 


\begin{tabular}{|c|c|c|}
\hline Contenido & LOU & LAU \\
\hline $\begin{array}{l}\text { Servicio a la } \\
\text { sociedad }\end{array}$ & Art. 1, puntos b) y c) & Art. 3, puntos c) y h) \\
\hline Calidad & Art. 31 , puntos a) y c) & $\begin{array}{l}\text { Art. } 58 \\
\text { Art. } 76 \text {, puntos } 1,2 \text { y } 3\end{array}$ \\
\hline Docencia & Art. 33 , puntos 1,2 y 3 & $\begin{array}{l}\text { Art. } 1 \text {, punto f) } \\
\text { Art. } 53 \text {, punto } 1\end{array}$ \\
\hline
\end{tabular}

Tabla 1: Marco legal para el desarrollo de la labor docente en Andalucía (tabla adaptada de [1]).

No obstante, no nos centraremos en los aspectos legales (¡menos mal!), dejando esta tarea para realización de los interesados (si los hubiere). Desde el punto de vista más aplicado, cuando se nos asigna una nueva asignatura nuestra labor sería obtener la memoria del título de grado verificada positivamente por la ANECA/AGAE. Recordar que ahora los títulos impartidos en los nuevos grados han sido previamente aprobados por las agencias de acreditación mediante el programa VERIFICA [10]. En esta memoria, buscaremos la asignatura que vamos a impartir y tendremos la información básica sobre contenidos, planificación, métodos de evaluación, etc. Los títulos verificados en la Universidad de Granada están disponibles para su consulta en [8]. Notemos que la información ahora disponible sobre qué impartir es ahora mucho más detallado que lo que había en los descriptores del BOE en la LRU. Como consecuencia, estamos mejor guiados y a la vez mucho más coartados en nuestra libertad de cátedra.

Si por algún motivo los títulos de grado hubieran sido desarrollados con mucha rapidez y por personal no correctamente formado en el proceso de adaptación al EEES, la información relativa a los contenidos y metodología de la materia a impartir podrían nos ser los más adecuados. Bueno, hay que vivir con ello y saber superarlo. Notemos que la ANECA ha establecido mecanismos de control (programa Monitor) para realizar un seguimiento del título oficial para comprobar su correcta implantación y resultados. Asociado al mismo cada titulación tendrá responsables encargados de supervisar estos factores (esperemos que para bien) y, con suerte, podrán realizarse modificaciones de los contenidos de la memorias de grado con objeto de mejorarlas.

A partir de aquí, el proceso/tarea a realizar sería la de concretar los contenidos, metodologías docentes y de evaluación, actividades y planificación de la asignatura a impartir.

Por ejemplo, a nivel de contenidos tenemos que pensar en:

- Los descriptores oficiales;

- La acción profesional;

- Las exigencias del contexto: a) institucional (estructura organizativa en la que se inscribe la propuesta); b) socio-profesional (finalidad formativa); c) 
curricular (interdisciplinaridad); y d) psicosocial (características de los destinatarios);

- Los estudios de competencias;

- El ámbito estatal y autonómico;

- El propio proceso de desarrollo profesional

- Las respuestas a preguntas de contenido como: ¿Cuántos temas pongo?, ¿Qué puede aportar?, ¿Qué temas son los fundamentales?

- Si vendrán profesionales a explicar el tema.

- Las conexiones con otras asignaturas.

- En qué tipo aulas trabajaremos y de qué medios dispondremos.

- Qué método usaremos para cada tema, por ejemplo ¿en cuáles talleres, cuáles magistral, cuáles indagación?

- El hilo conductor de los temas, ¿a qué interés responde?

Y a nivel de evaluación tenemos:

- Los criterios e instrumentos de evaluación: Evaluar por carpetas (portfolio), exámenes de preguntas cortas, largas o tipo ensayo, pruebas objetivas, trabajos opcionales, por contratos, mapa conceptual, defensa oral o escrita de un libro...

- Las alternativas a los que no aprueban: lecturas, tutoría

- El plan B para alumnos Erasmus, Séneca y de incompatibilidades horarias

- Los objetos de la evaluación que son: aprendizaje, programa de la asignatura, acción práctica del profesor

Como resumen, notemos que todos estos aspectos implican no sólo un profundo conocimiento técnico de la materia a impartir, sino además amplios conocimientos y recursos en el ámbito pedagógico y docente así como de los recursos que ofrece la institución universitaria en que trabajemos. De nuevo muchos aspectos fuera totalmente del alcance de este trabajo pero que esperamos sean abordados por los profesores de la Universidad en pro de una mejora de la docencia en las aulas.

\section{Si no doy la lección magistral, ¿cómo lo hago?}

El hecho de que el proceso de enseñanza-aprendizaje esté centrado en el estudiante no quiere decir que la lección magistral no sea un método docente válido (¡no nos asustemos!). La reforma educativa promueve que el docente aplique distintas metodologías docentes, eligiendo la más apropiada para los distintos contenidos y actividades de la asignatura, sin embargo, la parte magistral (convenientemente adaptada), sigue siendo un recurso válido.

Conviene hacer hincapié en algunos aspectos clave para que la reforma pueda ser todo un éxito. Tanto el profesorado como el alumnado deben estar concienciados en los cambios que trae la reforma, y el nuevo papel que cada uno debe desempeñar. El profesor debe estar dispuesto a cambiar su esquema de trabajo y convertirse en orientador o facilitador (dejar de ser un ponente), pues ahora el alumno es el protagonista de su propio aprendizaje, por lo que también habría que hacer un 
esfuerzo en comunicarles a los alumnos cuál va a ser su papel, antes de comenzar los estudios universitarios.

Entre los cambios que se esperan del profesorado podemos destacar la variedad metodológica, para lo que pasamos a comentar algunas metodologías o estrategias alternativas a la lección magistral que tradicionalmente (con sus ventajas e inconvenientes) se ha venido impartiendo en el ámbito universitario, y que está principalmente orientada a la memorización, más que a la comprensión (ver Figura 1) y consultase [1] para más detalles. No obstante, la lección magistral permite la introducción de elementos dinamizadores durante su desarrollo, desde preguntas a debatir con los estudiantes, inclusión de pequeños ejercicios para los alumnos, a sopas de letras y jeroglíficos que rompen con la monotonía del discurso.
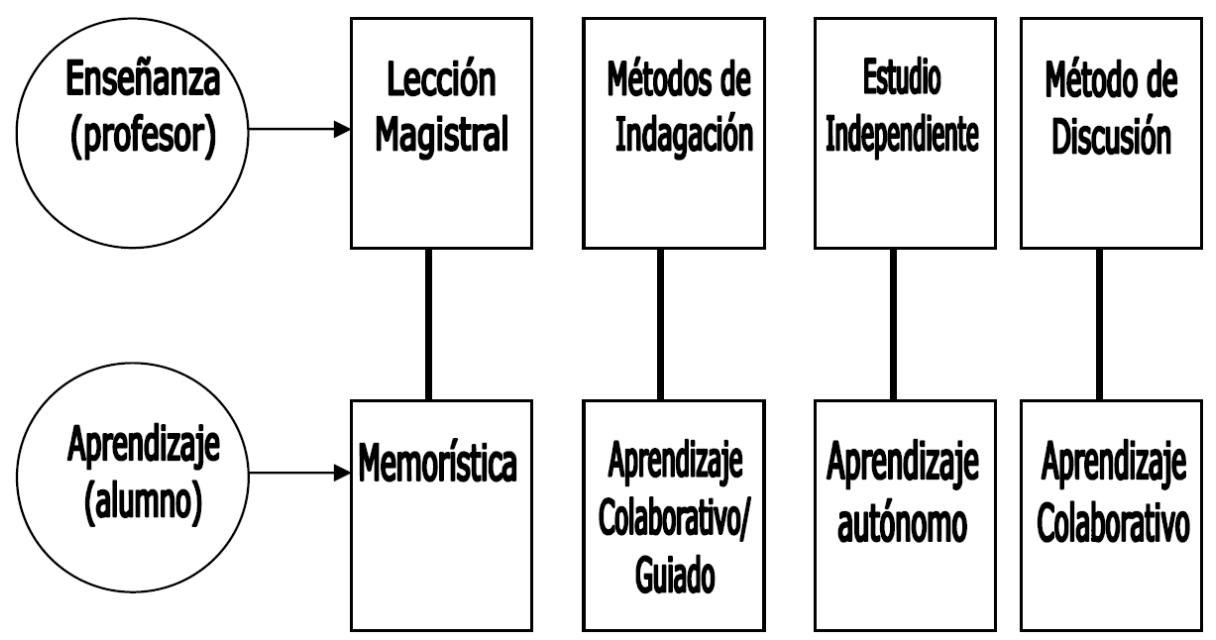

Figura 1: Relación entre los métodos de enseñanza y el tipo de aprendizaje por parte del alumno (figura adaptada de [1]).

En el caso de los métodos de indagación el alumno elabora sus conocimientos induciendo o deduciendo reglas a partir de datos o ejemplos que el profesor presenta. Se conoce también como aprendizaje basado en problemas, y el papel del profesor es el de guía en el proceso de aprendizaje, cerciorándose de su consecución. Una actividad dinamizadora para esta metodología es la elaboración de mapas conceptuales por parte de los alumnos, que permite constatar la correcta identificación y relación entre los conceptos clave. Éste es, principalmente, el paradigma que promueve el EEES.

Otra de las novedades que introduce la reforma es la importancia que se da al aprendizaje autónomo por parte del estudiante, mediante el estudio independiente. El estudiante es responsable de planificar, desarrollar y evaluar su aprendizaje, permitiéndole aprender a un ritmo personal y adecuado a sus condiciones e intereses. Para el buen desarrollo se hace necesario disponer de una buena biblioteca/hemeroteca, acceso a bases de datos e internet y lugares adecuados para el desarrollo del trabajo personal. Además, el alumnado debe estar preparado en técnicas de trabajo individual y el profesorado debe dedicar tiempo en clases y tutorías a orientar y supervisar este tipo de tareas individuales. 
También podemos usar este enfoque basado en aprendizaje autónomo para eliminar contenidos de la lección magistral. Cuando el temario lo permita, podemos indicar en la guía didáctica del alumno que un tema concreto ha de ser estudiado de forma autónoma. Ello, junto con una guía de trabajo autónomo al respecto que, incrementalmente, guíe al alumno en el proceso de aprendizaje y una bibliografía detallada, deberá permitir entender a un alumno un tema por sí mismo. Posteriormente en clase podemos debatir y aclarar las dudas del mismo. Esto tiene como ventaja al profesor que nos permite "no hacer las transparencias" de ese tema. Como contrapartida el diseño de la guía de trabajo autónomo de este tema requiere tanto o más esfuerzo como el de hacer las transparencias. En lo referente al alumno corremos el riesgo que directamente no realice la actividad pero, ¿Cuántas veces un alumno viene a clase y no atiende? Está sólo de cuerpo presente. Este método permite dar dinamismo a las clases y que cada alumno aprenda a su ritmo. Por supuesto tiene inconvenientes y probablemente no es válido para algunos aspectos del temario de nuestras asignaturas pero de nuevo es un recurso didáctico más que podemos explorar.

Un elemento para promover la reflexión, autoevaluación y el análisis crítico por parte del alumno es el portafolio. Podemos definir un portafolio como una colección de los logros individuales del alumno junto a la evaluación del proceso, las estrategias aplicadas, y el análisis sobre las experiencias de aprendizaje, por lo que es mucho más que una colección de tareas realizadas. Notemos que esta carpeta de actividades tiene un doble objetivo. Por una parte de ella podemos extraer las actividades que serán objeto de evaluación del estudiante (no necesariamente todas las actividades promovidas deben ser usadas para la evaluación del mismo). De otra parte puede ser un instrumento más para mejorar el aprendizaje del alumno.

Un gran problema que se encuentra el profesor es el relativo a que algunas tareas no son realizables debido al alto número de estudiantes matriculados (para un profesor que trabaja no más del número de horas exigido en su contrato). Las estrategias de trabajo en grupo pueden ser una herramienta para lidiar con ello. Puesto que en los títulos de grado se han definido actividades para grupo grande (toda la clase) y para grupo pequeño (típicamente lo que eran grupos de prácticas en titulaciones TIC), el tipo de actividades a realizar deberá ajustarse al tipo de grupo con el que contemos en cada momento.

Estas estrategias dan un papel más relevante al alumnado y podemos englobarlas bajo el método de discusión. Desarrollan el pensamiento crítico, las habilidades sociales e impulsa la independencia de juicio. En contra, requiere una buena planificación para evitar conflictos de grupo, el trabajo es más lento y hay que cuidar el tamaño del grupo de trabajo para que sea operativo (por ejemplo, elegir un número impar de miembros para evitar empates en la toma de decisiones que paralicen el trabajo). Dependiendo del tamaño de los grupos que realicemos y el tiempo que se le pueda/quiera dedicar a este tipo de actividades, podemos encontrar una amplia variedad de estrategias, entre las que destacamos el debate, el 'role playing', el foro, la mesa redonda, el simposio, el panel o la conferencia, especialmente destinadas a grandes grupos de participantes. Para grupos medianos podemos trabajar la lluvia de ideas o el grupo T. En grupos pequeños podemos aplicar técnicas más específicas como Phillips 6/6 (grupos de 6 personas discutiendo un problema durante 6 minutos), el cuchicheo (discusión de un problema en voz baja por parejas), la entrevista o el 
mantel de papel (papel de grandes dimensiones en el que cada alumno o sub-grupo escribe sus impresiones o decisiones sobre un tema concreto).

La aplicación de unos métodos u otros exige, en diferentes momentos del proceso, la organización de los alumnos en grupos de distinto tamaño. La lección magistral está dirigida al gran grupo, todos los alumnos de una clase; los métodos de discusión exigen que los grupos de trabajo sean más pequeños; en el caso del aprendizaje autónomo el alumno debe trabajar solo mientras que los métodos de indagación permiten diferentes configuraciones. Otra exigencia muy importante es la planificación, que debe estar presente en todos los aspectos que intervienen en el proceso de enseñanza-aprendizaje, desde los contenidos, metodologías y estrategias didácticas y de evaluación, hasta las tutorías [7].

Toda esta marabunta de nuevas técnicas y métodos nos llevan a otra idea, ¿cómo evaluar de forma eficaz todo el trabajo que realizan los estudiantes sin que suponga un conflicto con el resto de encargos que tiene el profesorado? En el EEES se apuesta firmemente por la evaluación continua (el $60 \%$ de la calificación debería estar fijada antes del examen), lo que puede llevarnos a una dedicación prácticamente por completo a la corrección de trabajos, supervisión de tareas, etc. Algunas ideas que pueden facilitarnos el trabajo serían la de (a) incorporar la evaluación entre iguales, en la que los alumnos evalúan a sus propios compañeros de forma responsable mediante técnicas como la rúbrica, la lista de control, o la escala de valoración, (b) definir tareas de autoevaluación o incluso co-evaluación, en la que la evaluación se establece como un acuerdo al que se llega entre profesor y alumno en una entrevista (c) usa de las herramientas TIC para automatizar el proceso de evaluación continua (ver por ejemplo [16]).

Notemos que un problema típico que se indica con el tema de la evaluación entre iguales es la de objetividad o capacidad de un alumno para evaluar al compañero. Si bien es necesario realizar una planificación adecuada por parte del profesor y dejar muy claro a los alumnos los criterios de evaluación (posiblemente consensuados con ellos), la experiencia dice que generalmente es un método que funciona muy bien. Además siempre podemos utilizar técnicas disuasorias (si algún alumno protesta reevaluamos nosotros y, si la nota puesta por el compañero no es acorde a los objetivos pactados, podemos puntuar negativamente al estudiante que ha hecho una evaluación incorrecta). Como conclusión operativa, o tenemos un número pequeño de alumnos (como en optativas de los últimos cursos) y corregimos todo nosotros, o podemos optar por corregir un número ingente de trabajos/actividades, o seguimos con clases Magistrales. Como mejor alternativa, os animamos a probar estas técnicas de evaluación entre iguales y a sorprenderos de su utilidad.

Otro elemento que interviene en el proceso de enseñanza-aprendizaje que, sin ser nuevo, adquiere mucha importancia en el EEES es la tutoría. Podemos decir que la tutoría se reinventa, deja de ser un tiempo para la mera consulta de dudas por parte del estudiante a ser un nuevo espacio temporal para el que podemos planificar actividades de seguimiento, de orientación o de cualquier tipo, tanto individuales como de grupo. Todo ello ha dado lugar incluso a proyectos específicos para mejora de las tutorías, los conocidos como planes de acción tutorial o PAT. Para más información ver por ejemplo el material disponible en [15].

Finalmente toda esta metodología debe apoyarse en el uso de las nuevas herramientas docente que, principalmente de la mano de las TIC, están disponibles. 
blogs, plataformas docentes virtuales, herramientas de consulta, material para presentaciones, etc. Todas estas herramientas deben conocerse y manejarse con soltura para, de esta forma, mejorar la comunicación entre profesor-alumno, hacer los contenidos más atractivos $\mathrm{y}$, en resumen, facilitar el proceso de aprendizaje del alumno.

\section{Resumen y conclusiones}

Esperamos que en este breve espacio hayamos podido describir, de forma básica y clara qué requiere de nosotros el proceso de adaptación al EEES y, con un poco de suerte, motivar al lector a profundizar en los temas aquí descritos. Como efecto adicional, esperamos que aquellos compañeros que no tenían claro cómo adaptar sus materias en este nuevo marco puedan, al menos, cumplir con la normativa vigente y que sus asignaturas sean "un poquito más a la Bolonia" y, aunque no cambien drásticamente su metodología docente, sí puedan dar pasos en la dirección que las instituciones y la política nos demandan.

Dicho todo esto, permitidnos un espacio para la reflexión personal. Surge la duda de si este nuevo enfoque promulgado por el EEES es el medio de mejorar la calidad de la docencia universitaria. Según se desprende de algunos trabajos como [6] y [13], muchos de los elementos son positivos (aprendizaje basado en el estudiante, aumentar nuestras herramientas metodológicas y docentes, etc...). No obstante, surgen otras cuestiones más asociadas al día a día, ¿proporciona el marco institucional los medios y la formación necesaria para aplicar estos cambios? Como es bien sabido, estos nuevos enfoques requieren un trabajo muy intensivo del profesor y son difíciles de llevar a cabo con grupos grandes. Además, ¿qué tanto por ciento de los profesores universitarios ha recibido formación para este cambio docente? La realidad dice que los grupos actuales son grandes y que la mayoría de los profesores (y alumnos) no tienen la formación adecuada para afrontar este cambio de enfoque docente.

En nuestra opinión, la filosofía actual basada en hacer cambios en la que nos hallamos sumidos, que muchas veces consiste simplemente en llamar con nombre distinto a las cosas y cuyo principal requisito es que sea a 'coste 0 ', tiene altísimas probabilidades de fracaso. Aunque de esta forma las estadísticas arrojen resultados positivos, el problema de base que consiste en tener universitarios que reciban la mejor formación posible seguirá sin solución. Si la docencia, y la universitaria en particular, es tan importante, ¿por qué no se apuesta firmemente por ella? ¿Por qué no se favorece la promoción del profesor que demuestra un alto grado de competencia en aspectos docentes, mientras que la investigación es el elemento principal que determina la promoción? Todo esto sólo puede salvarse (al menos parcialmente) apelando a la profesionalidad del cuerpo de los docentes universitarios que, de forma gratuita y en sus "ratos libres", deberán realizar un cambio mucho más profundo que el mero cambio terminológico.

Para concluir, un último aspecto a considerar es que el uso de las fichas Verifica y la obligación de usar esta metodología del EEES, no necesariamente es el camino hacia una docencia de calidad. Como indica [13], los mejores profesores son los que, de forma flexible y con gran nivel de conocimiento de la materia que imparten, son 
capaces de adaptarse a los alumnos e innovar. Tal y como vemos la reforma, parece que todo el profesorado debe andar prácticamente por los mismo itinerarios, seguir las mismas metodologías e impartir unos contenidos y/o planificación que en muchos casos no soportarían una revisión profunda de los mismos. Con el EEES se ha pretendido forzar la mejora de la docencia a coste de perder, en buena parte, aspectos relativos a la libertad de cátedra. Como consecuencia, se nivela al profesorado, obligando al profesor menos preocupado por aspectos docentes a enredarse en mil y una técnicas nuevas. Por otra parte, al profesor "excelente", se le impone un corsé que puede llegar a dificultar su faceta innovadora.

En el marco de la Universidad, es precisamente la singularidad de sus individuos y su complementariedad lo que le aporta un valor diferencial a todo el conjunto. El papel de las instituciones debería permitir mejorar la calidad de la educación, motivando (u obligando) al profesorado que lo requiera pero dejando libertad para el ejercicio de los "buenos" profesores, (cualesquiera que estos sean, tema no fácil de determinar). Durante estos años de adaptación veremos cómo el EEES afecta a unos y a otros y, en todo caso, apelaremos al sentido común de la comunidad universitaria para que, pese a todo, el resultado final sea satisfactorio.

\section{Referencias}

[1] Curso de Iniciación a la Docencia Universitaria ( $3^{\mathrm{a}}$ Edición), Múltiples autores. Universidad de Granada 2010-2011. Material e información disponible en: http://calidad.ugr.es/pages/secretariados/form apoyo calidad/programa iniciacion docen cia/iniciacion3index

[2] Cursos de formación para el profesorado del Vicerrectorado para la garantía de la Calidad, Universidad de Granada:

http://calidad.ugr.es/pages/secretariados/form_apoyo_calidad/index\#_doku_programa_d e ayuda a la formacion

[3] Curso de Planificación de la docencia universitaria por competencias y elaboración de Guías Didácticas $\left(2^{\mathrm{a}} \mathrm{ed}\right)$,

http://calidad.ugr.es/pages/secretariados/form_apoyo_calidad/programa-de-formacionpermante/curso_planificacion/2edicion/guiasdidacticas

[4] Materiales resultantes del Curso de Planificación de la docencia universitaria por competencias y elaboración de Guías Didácticas ( $\left.2^{\mathrm{a}} \mathrm{ed}\right)$. Disponibles en http://calidad.ugr.es/pages/secretariados/form apoyo calidad/programa-de-formacionpermante/curso planificacion/2edicion/guiasdidacticas

[5] Proyecto Tuning, desde http://tuning.unideusto.org/tuningeu/, (2004)

[6] Zabalza Beraza, Miguel Ángel (2003) Competencias docentes del profesorado universitario, Calidades y desarrollo profesional. Narcea. Madrid.

[7] Zabalza,M.A. y Zabalza, M. (2010). Planificación de la docencia en la Universidad. Madrid: Narcea. 
[8] Relación de memorias de títulos de Grado verificadas positivamente por la ANECA/AGAE http://vicengp.ugr.es/pages/grados-verificados

[9] Juan Freire, Estrategias para la adaptación al Espacio Europeo de Educación Superior en titulaciones de Ciencias (Cáceres y Badajoz, 8 y 9 Marzo 2005). Plan de Formación Docente del Profesorado Universitario de la UEX. Cursos sobre Diseño de Planes Docentes. Organizado por la Oficina de Convergencia Europea de la Universidad de Extremadura.

[10] Agencia Nacional de Evaluación de la Calidad y Acreditación ANECA: http://www.aneca.es/

[11] Libros blancos: http://www.aneca.es/Documentos-y-publicaciones/Libros-blancos2

[12] Riesgo, M., El enfoque por competencias en el EEES y sus implicaciones en la enseñanza y el aprendizaje, Tendencias pedagógicas, 13, pp. 79-105, (2008).

[13] Aristimuño, A., Las competencias en la educación superior: ¿demonio u oportunidad? Departamento de Educación, Universidad Católica de Uruguay, Uruguay (2004).

[14] Bain, K., Lo que hacen los mejores profesores ( $\left.2^{\mathrm{a}} \mathrm{Ed}\right)$, Universitat de Valencia, Valencia, España, (2007).

[15] Ejemplos PAT UGR: http://www.ugr.es/ vic plan/formacion/ato/index.html

[16] Romero, S. y Guillén, A., Evaluación y autoevaluación continuas automatizadas mediante herramientas de software libre. Congreso de Docencia Universitaria, Vigo (2009). 
Enseñanza y Aprendizaje de Ingeniería de Computadores, Número 1, 2011 